\title{
Author index (Volume 11)
}

Adimurthi, Ghoshal, S. S.

\& Gowda, G. D. V., Optimal controllability for scalar conservation laws with convex flux

Antonelli, P. \& Weishäupl, R. M., Asymptotic behavior of nonlinear Schrödinger systems with linear coupling

Beceanu, M., $A$ center-stable manifold for the energy-critical wave equation in $\mathbb{R}^{3}$ in the symmetric setting

Benjelloun, S., Desvillettes, L. \& Moussa, A., Existence theory for the kinetic-fluid coupling when small droplets are treated as part of the fluid

Bianchini, S. \& Modena, S., On a quadratic functional for scalar conservation laws

Blokhin, A. M. \& Tkachev,

D. L., Stability of a supersonic flow over a wedge containing a weak shock wave satisfying the Lopatinski condition

Bourdarias, C., Gisclon, M. \& Junca, S., Fractional $B V$ spaces and applications to scalar conservation laws

Chapiro, G., Marchesin, D. \& Schecter, S., Combustion waves and Riemann solutions in light porous foam

Chiodaroli, E., A counterexample to well-posedness of entropy

solutions to the

compressible Euler

system

3 (2014) 493

3 (2014) 477

Choudhury, A. P., Joseph, K. T. \& Sahoo, M. R., Spherically symmetric solutions of multidimensional zero-pressure gas 3 (2014) 437

1 (2014) 109 2 (2014) 355

2 (2014) 215

4 (2014) 655

2 (2014) 295 dynamics system

Contreras, A. \& Pelinovsky, D., Stability of multi-solitons in

the cubic NLS

equation

Coulombel, J.-F. \&

Williams, M.,

Amplification of pulses in nonlinear geometric optics

Deng, S., Wang, W. \& Yu, S.-H., Bifurcation on boundary data for linear broadwell model with conservative boundary condition

3 (2014) 603

Desvillettes, L., see

Benjelloun, S.

Ghoshal, S. S., see

Adimurthi

Gisclon, M., see

Bourdarias, C

Gowda, G. D. V., see

Adimurthi

2 (2014) 269

2 (2014) 329

4 (2014) 749

1 (2014) 109

3 (2014) 477

4 (2014) 655

Joseph, K. T., see Choudhury, A. P.

Junca, S., see Bourdarias, C.

2 (2014) 269

4 (2014) 655

Kawashima, S., see Mori, N.

1 (2014) 135

Kong, D.-X. \& Wang, J., Einstein's hyperbolic geometric flow
3 (2014) 477 
Kong, D.-X., Wei, C.-H. \& Zhang, Q., Formation of singularities in

one-dimensional Chaplygin gas

3 (2014) 521

Lukáčová-Medvid'ová, M. \& Sfakianakis, N., Entropy dissipation of moving mesh adaptation

Marchesin, D., see Chapiro, G.

Modena, S., see Bianchini, $\mathrm{S}$.

Mori, N. \& Kawashima, S., Decay property for the Timoshenko system with Fourier's type heat conduction

Moussa, A., see Benjelloun, $\mathrm{S}$.

Nishitani, T., Local and microlocal cauchy problem for non-effectively hyperbolic operators

Oh, S.-J., Gauge choice for the Yang-Mills equations using the Yang-Mills heat flow and local well-posedness in $H^{1}$

Pelinovsky, D., see Contreras, A.

Peralta, G. \& Propst, G., Local well-posedness of a class of hyperbolic PDE-ODE systems on a bounded interval

Perepelitsa, M., A note on strong solutions to the variational kinetic

3 (2014) 633

2 (2014) 295

2 (2014) 355

1 (2014) 135

1 (2014) 109

1 (2014) 185

1 (2014) 1

2 (2014) 329 equation for scalar

conservation laws

3 (2014) 621

Propst, G., see Peralta, G.

4 (2014) 705

Sahoo, M. R., see

Choudhury, A. P.

2 (2014) 269

Schecter, S., see Chapiro, $\mathrm{G}$.

2 (2014) 295

Sfakianakis, N., see

Lukáčová-Medvid'ová, M.

3 (2014) 633

Shao, A., New tensorial estimates in Besov spaces for time-dependent $(2+1)$-dimensional problems

4 (2014) 821

Tkachev, D. L., see

Blokhin, A. M.

Wakasugi, Y., On diffusion phenomena for the linear wave equation with space-dependent damping

4 (2014) 795

2 (2014) 249

Wang, J., see Kong, D.-X.

Wang, Q., Causal geometry of rough einstein

2 (2014) 215 CMCSH spacetime

Wang, W., see Deng, S.

Wei, C.-H., see Kong, D.-X.

Weishäupl, R. M., see Antonelli, P.

Williams, M., see Coulombel, J.-F.

$\mathrm{Xu}$, J., The well-posedness theory for Euler-Poisson fluids with non-zero heat conduction

4 (2014) 679

3 (2014) 603

Yu, S.-H., see Deng, S.

3 (2014) 521

3 (2014) 563

3 (2014) 603

3 (2014) 521

1 (2014) 159

4 (2014) 749

Zhang, Q., see Kong, D.-X. 